\title{
METALIZED SOFT POLYMERS FOR ELECTROMECHANICAL TRANSDUCERS ON GLASS SUBSTRATES
}

\author{
Boshen Liang ${ }^{12}$, Grim Keulemans ${ }^{1}$, Dominika Wysocka ${ }^{1}$, Lei Zhang ${ }^{1}$, Veronique Rochus ${ }^{1}$ \\ Tim Stakenborg ${ }^{1}$, Paul Heremans ${ }^{12}$, and David Cheyns ${ }^{1}$ \\ ${ }^{1}$ Imec, Leuven, Belgium \\ ${ }^{2}$ KU Leuven, Leuven, Belgium
}

\begin{abstract}
In this study, two different semiconductor-grade polymers are utilized to build up membrane-based electromechanical transducers. With three to four orders of magnitude lower modulus in comparison with silicon, the use of polymers as the vibrating membrane improves the mechanical output characteristics where larger vibration amplitude is needed.

Novel processing methods, including an excimer laser-aided wafer-to-water transferring technique, have been developed for the introduction of polymers into the standard cleanroom fabrication environment. Both piezoelectric and electrostatic transducers are fabricated on glass substrates and then characterized with laser dropper velocimetry. Comparison is made to exemplify the advantages and disadvantages of using polymers with varied dynamic specifications for different applications, where the compromise between fabrication robustness and device performance is needed.
\end{abstract}

\section{KEYWORDS}

CMOS Compatibility, Polymer, Electromechanical Transducer, Piezoelectric, Electrostatic

\section{INTRODUCTION}

Electrically modulated transducers have attracted great interest from both the research community and the commercial sector over the past several decades [1]. As shown in Fig.1, the electromechanical transducers convert electrical input signals to mechanical outputs via mechanisms such as piezoelectric and electrostatic couplings. Dominated by silicon-based technologies, the complexity and energy efficiency for such devices have been constantly improved over years [2]. However, for multiple emerging applications, represented by BioMEMS, microfluidics and ultrasonic transducers, larger vibrating amplitude beyond what silicon can achieve is preferred for meaningful operations [3]. Developed for decades, Polydimethylsiloxane (PDMS)-like materials enabled fast prototyping and have allowed to boost the actuating amplitude by orders of magnitude compared to silicon-based devices [4]. Nevertheless, the integration of PDMS-like polymers in industrial semiconductor environments is undesirable for mid and large-scale production. Furthermore, it has been a challenge to fabricate electrical and optical components ranging from basic metal contacts to sensors and processors directly on top of PDMS-like polymers due to their weak chemical resistance to commonly used solvents and acids in semiconductor and flat panel display (FPD) industries.

To address these issues, two different semiconductorgrade polymers are selected to build up electrically modulated transducers. Working as the vibrating membrane, the use of polymers, compared to pure siliconbased devices, provides not only the opportunity for a lower cost per production volume but also opens up the chances to bring in versatile thermal, mechanical, chemical and optical functionalities, such as transparency, flexibility and biocompatibility. What's more, compared with the PDMS-based approach that is largely employed in academic lab environments, the semiconductor-grade polymers used in the current study ensure the scalability of the technology to be adopted for highly integrated schemes of massive production. Using polymers with different dynamic properties, both piezoelectrically and electrostatically actuated prototype devices are fabricated and characterized to show the compatibility of the proposed materials and processing methods to semiconductor and flat panel display environments.

\section{MATERIAL CHOICE}

In this study, glass is used as the substrate for its high chemical resistance, high optical transparency and the potential to extend for mass production. A photopatternable negative resist is used to create the cavity below the vibrating membrane. For membrane polymers, 2 major factors, the mechanical dynamic property and the chemical resistance are evaluated to make sure the desired functions can be fulfilled, and the processing can be achieved with standard cleanroom facilities. Here, the dynamic property refers to the required mechanical deflection to external stimulus. The chemical resistance of polymers is checked for common solvents and acids used for metal patterning by photolithography, with representative examples as ethanol, butyl acetate, tetramethylammonium hydroxide, and phosphoric acid.

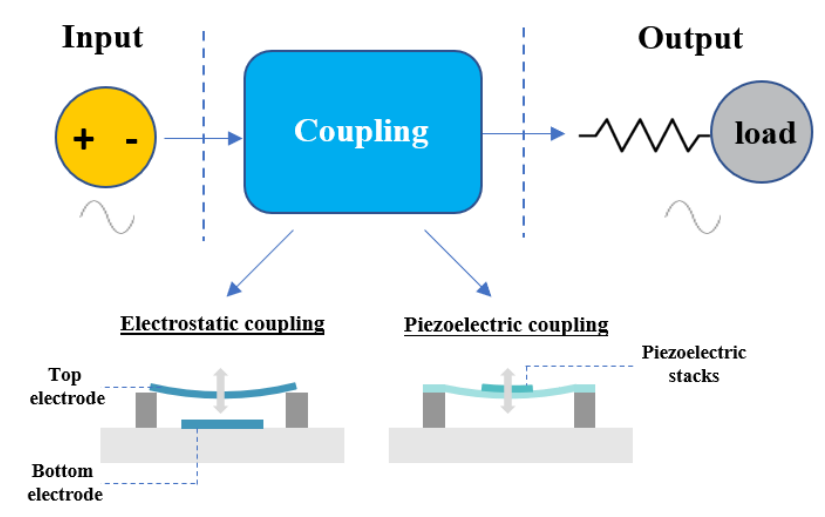

Fig. 1: Schematics of the micromachined polymer-based electromechanical transducers where electrical input is converted to mechanical vibration output via different mechanisms. 
Table 1: Material properties of two polymers used in this work compared to that of PDMS and Silicon [5]

\begin{tabular}{|c|c|c|c|c|c|c|}
\hline & $\begin{array}{c}\text { Young's } \\
\text { Modulus }\end{array}$ & $\begin{array}{c}\text { Solvent } \\
\text { resistance }\end{array}$ & $\begin{array}{c}\text { Acid } \\
\text { resistance }\end{array}$ & $\begin{array}{c}\text { Optical } \\
\text { transparency }\end{array}$ & $\begin{array}{c}\text { Gas } \\
\text { permeability }\end{array}$ & $\begin{array}{c}\text { Process } \\
\text { window }\end{array}$ \\
\hline Silicon & $\sim 180 \mathrm{GPa}$ & Strong & Strong & Bad & Small & Big \\
\hline PDMS & $\sim 1 \mathrm{MPa}$ & Weak & Weak & Good & Huge & Small \\
\hline Polyimide & $\sim 3 \mathrm{GPa}$ & Strong & Good & Moderate & Small & Big \\
\hline Polyisoprene & $\sim 100 \mathrm{MPa}$ & Moderate & Good & Moderate & Small & Moderate \\
\hline
\end{tabular}

\section{PI or PIP spinning}

6", Donor Glass

\section{Wafer level bonding and laser delamination}

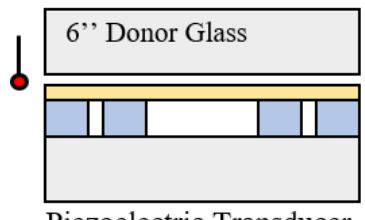

Piezoelectric Transducer

\section{Top part preparation}

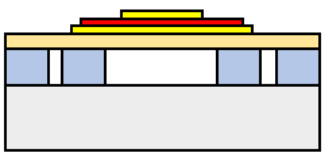

Piezoelectric Transducer

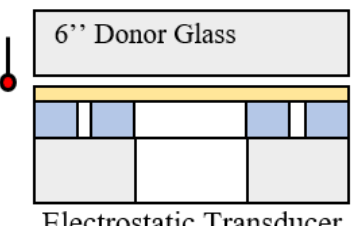

Electrostatic Transducer

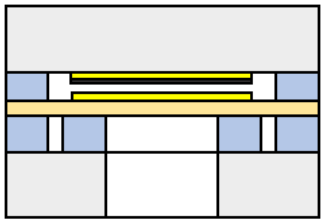

Electrostatic Transducer

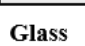

PI/PIP Photoresist PVDF
$\mathrm{SiN}_{\mathrm{x}}$ Metal contacts

Fig. 2: Fabrication process of the electromechanical transducers. The piezoelectric and electrostatic fabrication process flows share similarity concerning the preparation and transfer of polymers, while they are different for the preparation of the electrodes and other functional layers.

Two more factors, optical transparency and gas permeability are checked for completeness. It's noted that although the lower modulus is preferred for larger mechanical vibration amplitude, the weaker mechanical strength will generally degrade the fabrication robustness, such as thermal budget, chemical resistance and mechanical durability.

Based on the material selection criteria, for the current study polyimide (PI) is used for relatively smaller vibrating applications where harsh fabrication conditions can be tolerated, while for relatively larger vibrating applications polyisoprene (PIP) is used on the cost of narrowed processing window. Qualitative properties of PI and PIP polymers are measured and listed in Table 1 as compared to reported properties of silicon and PDMS [5].

\section{FABRICATION}

The fabrication process flows of the piezoelectric and electrostatic transducers share a great similarity, with the major differences lying in the different actuation methods and thus different ways of preparing the top electrodes and

other adjacent functional layers. As shown in Fig.2, firstly the polyimide or polyisoprene resin was spin-coated on a 6-inch donor glass wafer, and then transferred by excimer laser release (SUSS XBC300 Gen2 Debonder) to a 6", acceptor glass wafer with dry film photoresist layer prepatterned as the cavities and channels. For the electrostatic case, extra circular holes were perforated on the acceptor glass. The laser dose used for wafer-wafer delamination was optimized to be $160 \mathrm{~mJ} / \mathrm{cm}^{2}$ as the result of the balance between good debonding and minimum residue.

After membrane transferring, for piezoelectric transducers, $80 \mathrm{~nm}$ aluminum was deposited and patterned on top of PI as the bottom contact. P(VDF-TrFE) powder was dissolved in Methyl Ethyl Ketone and the solution was spin-coated on top of the wafer to form a $0.5 \mu \mathrm{m}$ piezoelectric layer, which was later cured in vacuum and $\mathrm{N}_{2}$ atmosphere. Another $80 \mathrm{~nm}$ aluminum layer was deposited and patterned as the top metal contact. For electrostatic transducers, due to the vulnerability of the weaker mechanical and chemical natures of polyisoprene, as well as the expected larger deflection, extra processing and design rules are applied to ensure the flexibility and reliability of the membrane. Specifically speaking, the 80 $\mathrm{nm}$ aluminum bottom electrode was elaborated designed into meander shapes, and the patterning of the metal with photolithography was optimized towards the lowest possible processing temperatures. Then, $80 \mathrm{~nm}$ aluminum counter electrode layer and $500 \mathrm{~nm} \mathrm{SiN}_{\mathrm{x}}$ dielectric layer, together with a $5 \mu \mathrm{m}$ spacer layer, is prepared on the top glass. Finally, the top and acceptor glasses are bonded together to form the final electrostatic transducer.

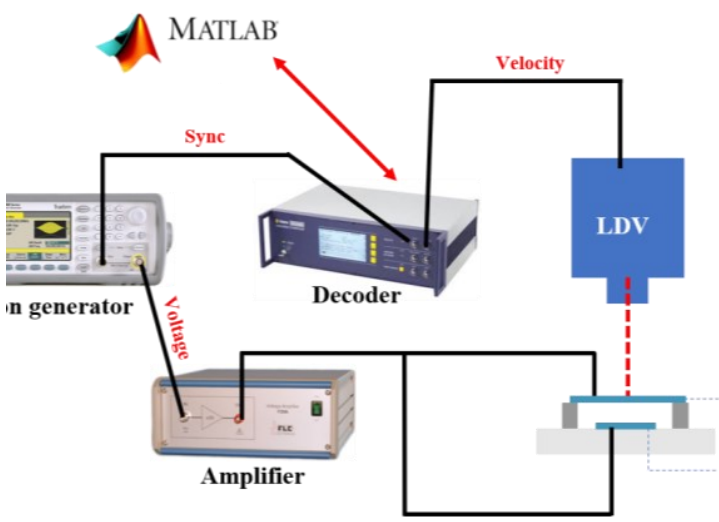

Fig. 3: Measurement setup for the piezoelectric and electrostatic transducers. An input signal is generator by a function generator and is sent to the electrodes of transducers after amplification. A LDV unit is used to capture the vibration, with a displacement decoder connected, together with MATLAB program to calculate the real-time vibrating amplitude. 


\section{RESULTS AND DISCUSSION}

\section{Measurement setup}

The measurement setup is shown in Fig.3. A function generator (Keysight 33500B) and a voltage amplifier (FLC electronics F20A) are used to apply electrical signals to drive the membrane. Laser Doppler vibrometer (LDV, Polytec MSA500) is used for membrane vibration characterization, with an amplitude decoder (2.5MHz DD900 Digital Displacement Decoder) used to convert the captured signal to vibrating amplitude by fast Fourier transform (FFT). A MATLAB program is used to automate data acquisition and post-processing.

\section{Piezoelectric transducer}

The piezoelectric transducer is successfully fabricated. As shown in Fig.4(a), occupying less than 1.5 $\mathrm{mm} \times 1 \mathrm{~mm}$ area on the glass wafer, each transducer unit consists of a suspended PI membrane, top and bottom electrodes, and fluid channels. The LDV laser beam is directed at the center of the top electrode for vibration characterization. The fluid channel is either emptied with only air or filled with water. At $1 \mathrm{~V}$ peak-to-peak voltage (Vpp), the LDV in high-frequency mode captured the resonance peak at $118 \mathrm{kHz}$ as in Fig. 4(b), which corresponds to the 1 st mode vibration in air. When water is filled to the underlying cavity via fluid channels, a clear shift of the resonant peak and amplitude is observed. Switched to the low-frequency mode of the LDV, the 1st

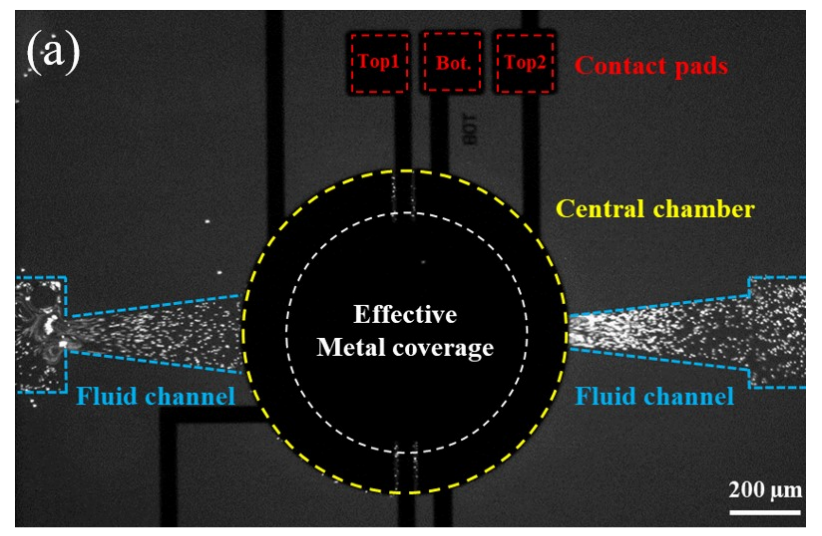

(b)

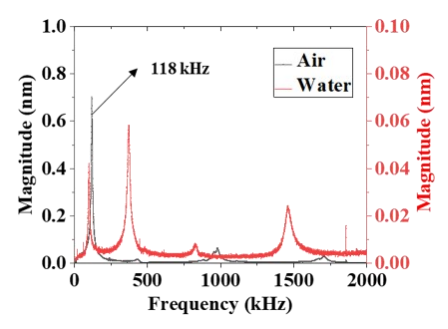

(c)

Fig. 4: Characterization of fabricated piezoelectric transducer. (a) The piezoelectric unit under microscope where fluorescent microparticles are added in liquid channel. (b) Frequency response of the piezoelectric transducer with or without fluid channel filled with water at high frequency mode. (c) $3 D$ reconstructed shape of the vibrating membrane measure at low frequency mode when the fluid channel is filled with water.

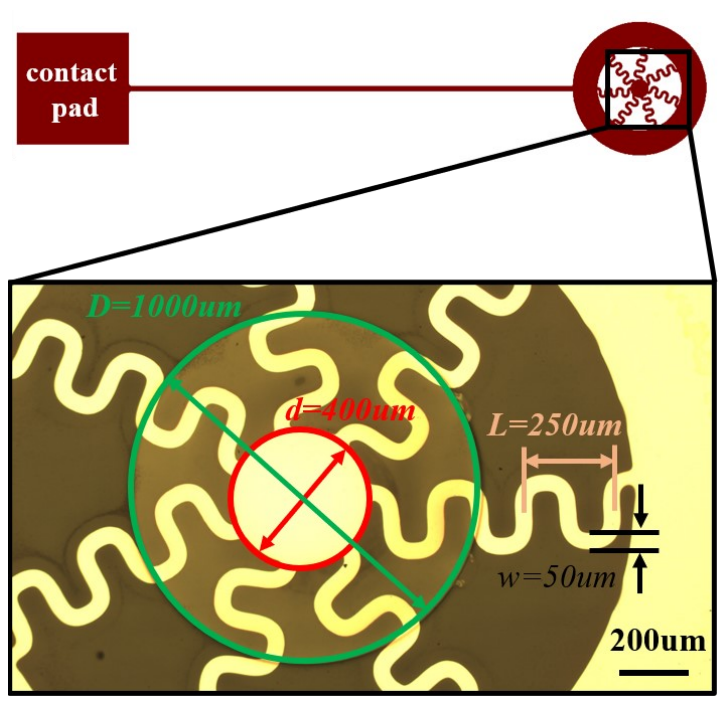

Fig. 5: Design parameters of the meander shaped electrodes on top of the soft PIP polymer.

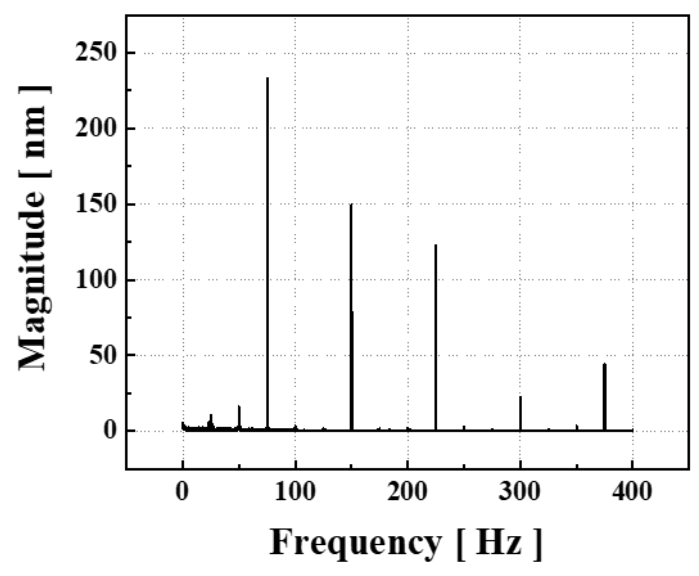

Fig. 6: Frequency response of the electrostatic transducer under AC sinusoidal driving voltage at $75 \mathrm{~Hz}, 100 \mathrm{Vpp}$.

mode frequency of the vibrating membrane after contacting with water changed to $4.4 \mathrm{kHz}$ and was reconstructed in 3D form as shown in Fig.4(c). Micron resolution particle image velocimetry (Micro-PIV) was used as another proof to show the damped vibrating of the membrane.

From the frequency drop from $118 \mathrm{kHz}$ in air to 4.4 $\mathrm{kHz}$ with water, based on the forced damped vibration theory [6], it can be deduced that the vibrating membrane is in near over-damping status. In such a case, the vibrating amplitude of the piezo-driven membrane $w$ can be modeled as

$$
w=\frac{F_{0}}{k} \sim \frac{E \times d_{31} \times M_{c}}{D}
$$

where $F_{0}$ is external force amplitude, $k$ the equivalent spring constant of the multi-layer membrane system, $E$ is the electric field applied to PVDF, $d_{31}$ is the PVDF piezoelectricity constant, $M_{c}$ is the electromechanical coupling efficiency [7], and $D$ is the flexural rigidity of the multi-layer membrane system. For a larger vibrating amplitude $w$, it can be shown from the formula that the 
Table2:Comparison of two polymer-based transducers

\begin{tabular}{|c|c|c|}
\hline & $\begin{array}{c}\text { Piezoelectric } \\
\text { Transducer on PI }\end{array}$ & $\begin{array}{c}\text { Electrostatic } \\
\text { Transducer on PIP }\end{array}$ \\
\hline $\begin{array}{c}\text { Polymer } \\
\text { modulus }\end{array}$ & $\sim 3 \mathrm{GPa}$ & $\sim 100 \mathrm{MPa}$ \\
\hline $\begin{array}{c}\text { Polymer } \\
\text { thickness }\end{array}$ & $15 \mu \mathrm{m}$ & $5 \mu \mathrm{m}$ \\
\hline $\begin{array}{c}1^{\text {st }} \text { mode air } \\
\text { Frequency }\end{array}$ & $>100 \mathrm{kHz}$ & $<1 \mathrm{kHz}$ \\
\hline $\begin{array}{c}\text { Metal } \\
\text { coverage }\end{array}$ & $76 \%$ & $16 \%$ \\
\hline $\begin{array}{c}\text { Amplitude } \\
\text { at 100 Vpp }\end{array}$ & $65 \mathrm{~nm}$ & $233 \mathrm{~nm}$ \\
\hline $\begin{array}{c}\text { Voltage } \\
\text { dependence }\end{array}$ & linear & quadratic \\
\hline
\end{tabular}

increase of applied voltage and thus the increase of electrical field $E$ will lead to a linear increase of the vibrating amplitude. Additionally, replacing PVDF with other piezoelectric materials for higher $d_{31}$, changing the thickness of PVDF and PI can be alternative effective methods to boost the vibrating amplitude.

\section{Electrostatic transducer}

Fig. 5 shows the fabricated electrostatic transducer, with key design parameters indicated on top of the microscope image. In the figure, $D$ is the diameter of the suspended membrane, $d$ is the diameter of the inner circular electrode. $L$ and $w$ stand for the length and the width of unit meander element that is then connected to the outside of the cavity by an annular ring and lead to the contact pad for probe statin via a long conducting path.

For the LDV measurement, the laser beam is directed at the center of the bottom electrode through the hole that is pre-perforated on the acceptor glass. As shown in Fig.6, when driving at a fixed frequency of $75 \mathrm{~Hz}$ at $100 \mathrm{Vpp}$, clear resonant peaks show at and on the multiples of the driving frequency. The vibrating amplitude at the $1^{\text {st }}$ resonant frequency is $233 \mathrm{~nm}$.

\section{Discussion}

The comparison of the piezoelectric and electrostatic transducers based on two different semiconductor grade polymers is summarized in Table 2 . It can be shown that there are distinct differences between the two devices originated from different polymers properties and driving mechanisms. Specifically speaking, the polyimide-based piezoelectric transducer, although featured with strong processing robustness thanks to the excellent mechanical and chemical properties of polyimide, has limited vibration amplitude due to the higher modulus of the polymer as well as the linear voltage dependence for the piezoelectric driving mechanism. What's more, the resonant frequency is in the relatively higher range. By comparison, featured with a much softer modulus, the polyisoprene-based electrostatic transducer vibrates at a much lower frequency, but achieves multiple times higher amplitude even with a lower effective metal coverage ratio. Thanks to its quadratic voltage dependence, the vibrating amplitude of the electrostatic transducers can be boosted even more by applying higher voltage up to $500 \mathrm{~V}$ before the breakdown of the $\mathrm{SiN}_{\mathrm{x}}$ dielectric layer. However, compared to polyimide, the polyisoprene polymer has less strong mechanical and chemical strengths, and thus extra design and fabrication rules, such as elaborate electrode design and lower processing temperature, have to be applied to make sure the soft polymer is well protected during all the fabrication processes.

\section{CONCLUSION}

Semiconductor-grade polymers of distinct properties are used to build-up piezoelectric and electrostatic transducers on glass substrates with cleanroom compatible processing methods. Compared to conventional siliconbased devices, the use of polymers enables larger vibrating amplitude thanks to their orders of magnitude lower softness. Tailored for different applications, a good balance between fabrication robustness and device performance should be considered when choosing proper polymer candidates and electro-mechanical coupling mechanisms.

\section{REFERENCES}

[1] K. L. Ekinci, "Electromechanical Transducers at the Nanoscale: Actuation and Sensing of Motion in Nanoelectromechanical Systems (NEMS)," Small, vol. 1, pp. 786-797, 2005.

[2] Z. Chang, "New Trends of the Development of Transducer Technology," J. Kunming Metall. Coll., p. 05, 2005.

[3] P. Abgrall and A. M. Gué, "Lab-on-chip technologies: Making a microfluidic network and coupling it into a complete microsystem A review," Journal of Micromechanics and Microengineering, vol. 17. p. R15, 2007.

[4] W. H. Grover, A. M. Skelley, C. N. Liu, E. T. Lagally, and R. A. Mathies, "Monolithic membrane valves and diaphragm pumps for practical large-scale integration into glass microfluidic devices," Sensors Actuators B Chem., vol. 89, no. 3, pp. 315-323, 2003.

[5] A. Mata, A. J. Fleischman, and S. Roy, "Characterization of Polydimethylsiloxane (PDMS) Properties for Biomedical Micro/Nanosystems," Biomed. Microdevices, vol. 7, no. 4, pp. 281-293, 2005.

[6] P. Howell, G. Kozyreff, and J. Ockendon, Applied solid mechanics. 2009.

[7] A. Ben Amar, H. Cao, and A. B. Kouki, "Modeling and process design optimization of a piezoelectric micromachined ultrasonic transducers (PMUT) using lumped elements parameters," Microsyst. Technol., vol. 23, no. 10, pp. 4659-4669, 2017.

\section{CONTACT}

*Boshen Liang, phone: +32-016-283-402;

Email: boshen.liang@imec.be 